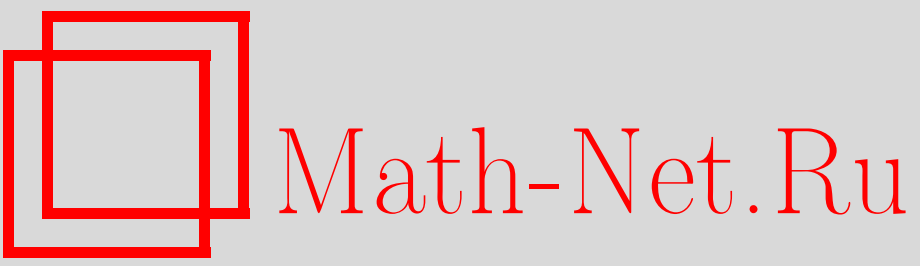

Д. Ван Дален, Колмогоров и Брауэр о конструктивной импликации и правило противоречия, УМH, 2004, том 59, выпуск 2, 53-64

DOI: https://doi.org/10.4213/rm717

Использование Общероссийского математического портала Math-Net.Ru подразумевает, что вы прочитали и согласны с пользовательским соглашением

http://www . mathnet.ru/rus/agreement

Параметры загрузки:

IP: 54.172 .240 .79

26 апреля 2023 г., 15:45:47 


\title{
КОЛМОГОРОВ И БРАУЭР О КОНСТРУКТИВНОЙ ИМПЛИКАЦИИ И ПРАВИЛО ПРОТИВОРЕЧИЯ
}

\author{
Д. ВАН ДАЛЕН
}

\begin{abstract}
В своей диссертации (1907) Брауэр высказал несколько замечаний по предмету, которьй стал в дальнейшем назьваться интуиционистской логикой. Его жесткая алгоритмическая точка зрения привела к отказу от принципа противоречия и, к сожалению, ко многим другим следствиям. В статье Колмогорова по логике 1925 года правило противоречия также отменено. Но в 1932 году, на основании "подразумеваемой интерпретации" (проблемы интерпретации), Колмогоров пришел к заключению о справедливости этого правила. Гейтингова интерпретация доказательств привела к тому же вьводу. Мы анализируем роль импликации и отрицания в принципе противоречия в трудах Брауэра, Колмогорова и Гейтинга. Мы утверждаем, что точка зрения Брауэра 1907 года слишком жестка для создания удовлетворительной логики, и он выходит за рамки минималњной логики в своем чтении импликации. Мы сравниваем перечисленные выше взгляды и заключаем, что проблема интерпретации и интерпретация доказательств, будучи правильно понятыми, допускают полную интуиционистскую логику.

Библиография: 35 названий
\end{abstract}

\section{СОДЕРЖАНИЕ}

Введение . . . . . .

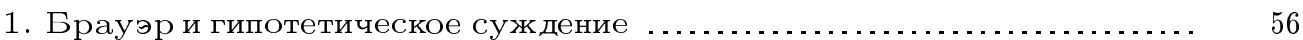

2. Принятие правила противоречия ................................ 59

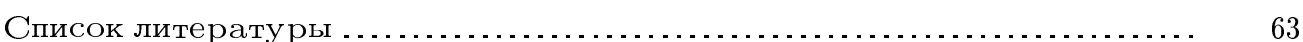

\section{Введение}

Колмогоров оставил свою печать на многих разделах математики, и он был во всех отношениях универсальньм математиком. В длинном списке проблем логика постоянно бросается в глаза. Колмогоров внес вклад в новую тему, не сушествовавшую в его время: в интуищионистскую логику. Его первая работа в этой области “О принципе tertium non datur*", была первьм примером успешной аксиоматизации неклассической логики. Эта статья предложила не только формализацию того, что сейчас известно как минимальное пропозициональное исчисление или минимальное исчисление

\footnotetext{
*Tertium non datur (лат.) - третьего не дано. - Прим. перев.
} 
высказьваний, но также и значительную часть металогики, а именно перевод Колмогорова, которьй предшествовал переводам Гёделя и Геншена. В своей второй статье “К толкованию интуиционистской логики" Колмогоров ввел семантику, естественно предназначенную для интуиционистской логики. Эта семантика была опубликована, грубо говоря, в то же время, что и гейтинговская интерпретация доказательств. Нетрудно видеть, что эти две интерпретации тесно связаны. Обе они основаны на идея Брауэра, которые во многом могут быть найдены в его диссертации 1907 года и в переписке с консультантом по его диссертации. По этой причине часто говорят о БГК-интерпретации.

Всестороннее изучение статей Колмогорова было проведено в большом числе работ. Не претендуя на полноту, мы отсылаем читателя, например, к [27], [29]-[31], [34], [35]. Общую информацию о БГК-интерпретации можно найти в [10], [12]-[15], [32]. В настояшей работе мы интересуемся главным образом статусом правила противоречия в конструктивных рассуждениях. Взгляды на это правило отцов-основателей интуиционистской логики, Колмогорова, Гливенко и Гейтинга, были различны. Поучительно предпринять попытку анализа их аргументов.

Конструктивная логика медленно прояснялась с течением лет. Первые следы можно найти в диссертации Брауэра (1907) и в его переписке со своим консультантом Кортевегом ${ }^{1}$. C самого начала Брауэр проводит различие между традиционной аристотелевой и логико-математической логическими практиками. Согласно Брауэру, традищионная логика развивается последовательными цепочками силлогизмов и применением принципов противоречия и исключенного третьего. Математический подход к логике состоит в конструктивной деятельности; начинаем с математической структуры, действуем на нее и, через несколько шагов, получаем новую структуру. Метафора, использованная Брауэром, вьплядела так: “уместить (математическое) здание в другое здание".

Терминология несколько неясная, но безусловно наводящая на определенные мысли. Вот пример: 8 является суммой двух нечетных простых чисел. Нужно построить здание для числа 8 и затем здание, состоящее из двух простых чисел, построить их сумму и встроить результат в здание для числа 8.

Мы выберем два простых числа, 3 и 5, построим сумму $3+5$ и проведем построение, сравнивающее эту сумму с 8. Сравнение оказьвается успешньм, и, следовательно, мы провели вложение данной структуры (здания) во вторую структуру. Здесь “умещение" означает, ввиду установленного факта, “тождественное совпадение”. Универсальное утверждение вроде “любое четное число является суммой двух нечетных простых чисел" приходит к вложению структуры всех четных чисел в структуру всех сумм двух нечетных чисел.

На самом деле, метафора о вложении в значительной степени сбивает с толку; например, во введенном выше мини-здании здание для числа 8 содержит больше, чем просто число 8. Это здание содержит также предыдущие натуральные числа, использованные при построении числа 8 . Здание для $3+5$ содержит еше больше, а именно также и оператор сложения для $3+n$ при $n \leqslant 5$. Поэтому вложение содержит больше подробностей, чем можно было ожидать вначале. Можно также допустить и главное здание всех натуральных чисел с богатым семейством операций и отношений.

\footnotetext{
${ }^{1}$ См. [5], издание 2001 г.
} 
Очевидно, мы здесь имеем дело с интерпретацией импликации. По существу импликация восходит к брауэрову понятию “скачка от цели к средствам". Чтобы объяснить это понятие, следовало бы рассмотреть брауэрово понятие "причинной последовательности". ${ }^{2}$ Мы опустим подробности и только отметим, что, согласно философским взглядам Брауэра, индивид (назьваемьй суббектом) наблюдает конечные последовательности событий (экспериментов, чувственных восприятий). Эти события могут находиться за пределами управления субъекта, но они могут быть так же хорошо сконструированы самим субъектом. Мы теперь предположим, что субъект может генерировать некоторую последовательность $e_{0}, e_{1}, e_{2}, \ldots, e_{n}$ и что $e_{n}$ является желаемым событием. Есть случаи, когда эта последовательность более или менее определена, т.е. если происходит $e_{0}$, то события $e_{1}, e_{2}, \ldots, e_{n}$ произойдут автоматически. Часто случается, что субъект не должен проходить через всю последовательность, чтобы добраться до $e_{n}$. Вместо этого оказывается, что он может осуществить промежуточное событие $e_{k}$ и затем автоматически продвигаться к желаемому результату $e_{n}$. Тогда конеи (иель) $e_{n}$ оказывается замененным средством $e_{k}$. Это событие $e_{k}$ может в свою очередь оказаться целью (концом) другой последовательности, для которой можно найти подходяшие "средства". И так далее. Это явление часто встречается в науке; можно хотеть доказать теорему и обнаружить, что "если я смогу доказать эту лемму, то ...".

Мы теперь находимся вблизи понятия импликации. В математике устанавливают, что "если $e$, то $e^{\prime \prime}$ ", выполняя промежуточные шаги, которые верно и точно приводят от $e$ к $e^{\prime}$. Это называется доказательством $e^{\prime}$ при данном $e$. Иначе говоря, у нас есть некоторьй алгоритм, который производит $e^{\prime}$, когда ему предоставляют $e$. Таким образом, мы можем эффективно породить единственную (или "какую-то") последовательность правильного типа. Здесь мы видим прототип доказательства - или проблему интерпретации.

Учитывая брауэровское понятие причинной последовательности и скачок от цели к средствам, мы оказываемся почти вьнуждены принять конструктивную импликацию, набросок которой дан выше.

Брауэр никогда не формализовьвал и даже словесно не формулировал интерпретацию доказательства, но из его немногих замечаний и из его практики можно понять, что он имел в виду.

В тридшатые годы и Гейтинг, и Колмогоров представили специальную интерпретацию интуиционистской логики. Довольно странно, что оба они представили формализации интуиционистской логики за несколько лет до этого: формализация Колмогорова была опубликована в 1925 году, а Гейтинга - в 1930-м. Можно предположить, что в это время они оба уже имели в виду некоторые интерпретации пропозициональных связок. Действительно, Гейтинг сообшил автору, что во время формализации он уже представлял себе (форму) доказательства интерпретации. Мы можем быть уверены, что Колмогоров был в том же положении. В конще концов, если хочется формализовать понятие, его нужно сначала понять.

Исторически, положение Гейтинга как студента Брауэра более или менее объясняет, как он формировал свои идеи. Интерес Колмогорова к конструктивной логике нельзя объяснить столь же просто. Как он получил свою информацию об интуицио-

\footnotetext{
${ }^{2}$ Cp. c [11].
} 
низме и его возможной логике? Известно, что контакт между Амстердамом и Москвой был: Александров и Урысон встречались с Брауэром в 1923 и 1924 году, и Александров и Брауэр тесно сотрудничали в годы, следовавшие за 1924-м. Хотя сам Александров не проявлял никакого интереса к интуищионизму, он вполне мог рассказать Колмогорову о конструктивизме Брауэра. В то время, когда Колмогоров начинал свои логические исследования, он знал сочинения [6]-[8], но, по-видимому, не [5]. Таким образом, Колмогоров пропустил отдельные замечания Брауэра о логике, которые показьвают в ретроспективе поразительное сходство с колмогоровским отказом от “принципа противоречия".

\section{1. Брауэр и гипотетическое суждение}

Гипотетические суждения имеют вид “если $A$, то $B$ ”, где не берется никаких обязательств по поводу истинности утверждения $A$. Например, в случае правила modus ponens, обязательство, можно сказать, встроено: " $A$ и $A \rightarrow B$ дают $B$ ". В обшем случае всякая информация подобного рода недоступна, и это не имеет значения, пока логика рассматривается как формальное исчисление. Хотелось бы, однако, дать основательную интерпретацию для $A \rightarrow B$. Традиционная логика апеллирует к значениям истинности: “ $A$ ложно или $B$ истинно”, но для конструктивной интерпретации это, очевидно, недостаточно. Именно поэтому Брауэр явным образом обсуждал этот вопрос в своей диссертации. Он критиковал общепринятый обычай допускать некоторую структуру для $A$ (или, на современном языке, модель) и рассуждать “гипотетически". Вместо предполагаемой структуры он требовал некоторую построенную структуру.

Колмогоров, в статье "О принципе tertium non datur" [25], видел, что перед ним та же проблема. Он не вполне специфицировал значение связок, но позаботился о том, чтобы придать смысл импликации:

"Значение символов $A \rightarrow B$ исчерпьвается тем, что убедивиись в истинности $A$, мы обязаны признать истинным и $B "$.

$\mathrm{C}$ этой точки зрения принцип противоречия $A \rightarrow(\neg A \rightarrow B)$ сомнителен, чтобы не сказать больше. На самом деле Колмогоров решил от него отказаться. Он рассуждал так: "Рассматриваемая теперь аксиома не имеет и не может иметь интуитивных оснований как утверждающая нечто о последствиях невозможного: мы обязаны признать $B$, если признали ложньм истинное суждение $A$ ". Ввиду более поздней колмогоровской “проблемы интерпретации" мы можем предположить, что у Колмогорова были возражения, подобные брауэровским. Возможно, однако, что эта формулировка способна указать путь, которьй стал известен как “логика релевантности”. Как кажется, упоминание “следствия" придает определенное правдоподобие этой точке зрения.

Следующее место в диссертации Брауэра, на которое до сих пор во многом не обращали внимания, проливает свет на брауэровский “конструктивньй” взгляд на логику: 
"Есть частный случай, в котором цеп силлогизмов имеет несколько иной характер, который кажется более близким к обычным логическим фигурам и который действительно кажется предполагаюшим гипотетическое суждение логики. Это происходит, когда некоторая структура внутри другой структуры определяется некоторым соотношением, в котором нельзя непосредственно распознать средства для его построения. Это вьглядит так, как будто в этом случае предполагается, что требуемая структура построена и что из этого предположения вьводится цепочка гипотетических суждений.

Но это - только видимость; то, что происходит в этом случае, состоит в следующем: начинаем построение системы, удовлетворяющей части требуемых соотношений, и пытаемся вывести из этих соотношений другие так, чтобы в результате вьведенные соотношения могли быть скомбинированы с еше неиспользованньми в систему условий, которая может служить отправной точкой для построения требуемых систем. Только с помошью этого построения мы показьваем, что условие действительно может быть вьполнено".

Этот отрывок непосредственно связан с интерпретацией импликации. Рассмотрим импликацию $A \rightarrow B$. Эвристика скачка от цели к средствам предполагает, что $A$ описывает математическое положение дел, скажем, структуру с ее соотношениями, постоянными, функциями и т. д. Как только $A$ реализована, мы знаем, как реализовать структуры для $B$ на основании некоторой конструкции. Теперь нет никакой необходимости в предварительных гарантиях, что в нашем распоряжении есть конструкция для $A$, так что в вышеприведенных строчках Брауэр указывает именно то, что, как только условия и спецификации для $A$ даны, мы пытаемся добавить информацию так, чтобы после определенного количества конструктивной деятельности мы действительно могли произвести построение $A$, удовлетворяющее спецификациям. Как только все это оказьвается вьполненным, мы можем заняться "импликацией"-построением для $B$, которое приводит к построению для $B$ и требуемому вложению структуры для $A$ в структуру для $B$.

Таким образом, структура для $A$ может быть недоопределенной, но должна быть возможность добавить спецификации так, чтобы ее можно было осуществить. Можно вспомнить старую практику в геометрии, где требуется построение некоторой фигуры, но некоторые параметры пропущены. Проводится анализ задачи и предоставляются, если это необходимо, дополнительные точки, прямые, окружности и тому подобное, пока построение не завершается. В качестве примера Брауэр указьвает построение совместной гармонической пары для двух коллинеарных пар точек. Это действительно можно сформулировать в виде импликации; если $A, B$ и $C, D$ - неразделяюшие пары, то имеется общая гармоническая пара $P, Q$.

Приведенная вьше процедура встречается также в обычной математической практике, что отражается в словах "не будет ограничением предположить ... "; это означает, что допустимо упростить задачу, предполагая, что "точки находятся на сфере", "функции дифференцируемы”, "пространство является метрическим" и т. д. Требуемая структура может быть получена из структуры со специальными свойствами.

$\mathrm{K}$ сожалению, замечания Брауэра о гипотетических суждениях довольно загадочны. Их можно толковать разными способами. Наиболее правдоподобньм прочтением вьглядит следующее: 
$(\alpha)$ Чтобы установить, что $A \rightarrow B$, нужно решить две задачи, (i) найти построение для (структуры, специфищированной) $A$, (ii) найти построение (для структуры, специфицированной) $B$, отправляясь от первого построения.

Мы оставили неявной ссылку на "вложение". На самом деле это вложение обычно молчаливо включается в построение для $B$.

Такое прочтение принимает во внимание замечания о расширении условий и, возможно, структуры. Они становятся, так сказать, методологическими.

Мы проиллюстрируем сказанное выше примером из Гейтинга [23]. Речь идет об импликации “если в десятичном разложении $\pi$ встречается последовательность из 100 нулей, то в нем также встречается последовательность из 99 нулей". 3 В брауэровской интерпретации 1907 года, нужно начать с построения последовательности 100 нулей в десятичном разложении числа $\pi$, и затем нужно указать построение для получения последовательности из 99 нулей для $\pi$. Второе построение действительно тривиально, но первое построение до сих пор не проведено. Таким образом, в строгой интерпретации, нельзя рассматривать эту импликацию как доказанную.

Другое, более вольное прочтение замечаний Брауэра было предложено в беседе Марком ван Аттеном. Перед тем, как обсуждать его, мы приведем еше несколько примеров.

Сначала заметим, что некоторое дополнительное замешательство вызьвают примеры, которые приводит Брауэр в своей диссертации. Он относит построение общей гармонической пары для двух пар коллинеарных точек, построения окружностей Аполлония и свойство единственности в теории груп Ли к примерам гипотетических суждений. Однако в двух геометрических примерах исходные структуры не вызывают сомнений, никаких дополнительных условий не нужно и т.д. Таким образом, бремя доказательства в этих случаях скорее лежит на втором построении (ii), чем на гипотетической структуре. Но сообщение верно и в этом случае: мы не предполагаем наличия двух (неразделяюших) пар, а можем их построить (тривиальным образом).

Рассмотрим частньй случай, в котором только построение структуры для $A$ сомнительно. При строгом прочтении брауэровского описания можно получить противоречивое множество условий, комбинируя все оставшиеся условия с уже использованными условиями, и, следовательно, структуры, как требует Брауэр, не получится. Поэтому вложение нельзя осушествить, что означает, что импликация не реализуется. Это находится в соответствии с методом "скачка от цели к средствам"; на самом деле нужно найти промежуточньй шаг, откуда можно достичь цель. Иньми словами, недостаточно иметь общий алгоритм, как достичь (структуры для) $B$ из (структуры для) $A$, но алгоритм должен быть успешно реализован.

Ясно, что подход Брауэра блокирует применение правила противоречия, но в немыслимо жестком виде: $A \rightarrow(\neg A \rightarrow A)$ было бы недопустимо, тогда как общее правило $A \rightarrow(B \rightarrow A)$ верно. Это притиворечит традищионной математической практике. На самом деле мы часто сталкиваемся с переопределенньми данными, и тогда приходится просто выбирать подходяший набор, позволяюший решить задачу.

Вот поучительньй пример: (1) если $n$ является степенью числа 6, а $m$ - степень числа 8, то п - четное.

\footnotetext{
${ }^{3}$ Последовательность 0123456789 была любимой последовательностью Брауэра [8], но эта последовательность десятичных знаков была недавно найдена в разложении числа $\pi$ [4].
} 
Работает следуюшее рассуждение: пусть $n$ - степень числа $6 ; 6$ делится на 2 , следовательно, $n$ делится на 2 и поэтому является четньм числом, что и требовалось доказать.

Заметим, что данные о числе $m$ вообще не использовались.

Осушествим теперь небольшое изменение: (2) если $n$ является степенью числа 6 и $n$ - степень числа 8, то $n$ - четное.

Мы можем просто повторить рассуждение для (1).

Однако с точки зрения строгого чтения Брауэра мы не можем построить требуемую структуру, т.е. такую, в которой $n$ является и степенью числа 6 , и степенью числа 8, и, следовательно, импликация не может быть реализована.

При обсуждении Марк ван Аттен заметил, что несколько более снисходительное чтение слов "в результате выведенные соотношения могли быть скомбинированы с еще неиспользованными" могло бы разрешить комбинировать вьведенные соотношения с подмножеством исходных условий. Это - необычная интерпретация "комбинирования". Например, если футбольная команда Соединенного Королевства создается комбинированием команд Англии, Шотландии и Уэльса, то группа из 33 игроков не нужна. Это прочтение могло бы заново допустить правило противоречия для людей с доброжелательным умонастроением. Остается неясньм, занимал ли сам Брауэр мягкую точку зрения. Менее строгое прочтение замечаний Брауэра имеет преимущество удаления или по крайней мере смягчения неестественных эффектов. В частности, $A \rightarrow(\neg A \rightarrow A)$ при этой интерпретации оказьвается правильным, как и $A \wedge \perp \rightarrow A$. Однако пример Гейтинга остается препятствием.

Итогом приведенных выше размышлений является то, что правило противоречия является сомнительньм, если не вообще неверным.

\section{2. Принятие правила противоречия}

В первой статье Гейтинга по интуиционистской логике объяснение импликации не разработано. В его формулировке:

Вообще говоря, вот что означает формула $A \rightarrow B$ : "если $A$ правильно, то В тоже правильно".

Без дальнейшей спецификации понятия “правильно" это разъясняет не больше, чем приведенное вьше объяснение Колмогорова. Гливенко, третий инициатор интуиционистской логики, использовал аналогичную формулировку: “формальная импликация $P \rightarrow Q$ имеет лишь тот смьсл, что 'если признается истинность $P$, то нужно признать истинность $Q^{\prime \prime}, 4$

Гейтинг в своей формализации явно принял принцип противоречия. Вот что следует за его объяснением смысла импликации $A \rightarrow B$ :

\footnotetext{
${ }^{4}[25],[20 ;$ c. 44$],[18]$.
} 
Возможен случай, когда после того как утверждение, что $A \rightarrow B$, удается доказать в специфицированном смысле, оказьвается, что $B$ верно всегда. Тогда, будучи однажды принятой, формула $A \rightarrow B$ должна оставаться верной; таким образом, мы должны придать смысл знаку $\rightarrow$ так, чтобы $A \rightarrow B$ продолжало быть верньм. То же самое можно заметить и в случае, когда в дальнейшем оказьвается, что $A$ всегда ложно. По этим причинам принимаются формулы

$$
2.14 \vdash B \rightarrow(A \rightarrow B) \text { и } 4.1 \quad \vdash \neg A \rightarrow(A \rightarrow B) .
$$

Что касается формулы 2.14, рассуждение верно, но, похоже, оправдание ключевого принципа 4.1 связано с тем же вопросом, которьй возник на мета-уровне. Кроме того, этот переход несколько сомнителен; он предполагает, что если у нас есть правильная импликация и если антецедент оказьвается ложным (абсурд), то импликация остается верной. Но это не покрьвает общий случай, в котором ничего не известно заранее об импликации. Мы можем согласиться, что требуется более детальное объяснение смысла импликации для оправдания принципа противоречия.

В 1932 году Колмогоров опубликовал полньй вариант своей “проблемы интерпретации" 5 . Здесь утверждения интерпретируются как задачи и конструктивная истинность $A$ превращается в "у нас есть решение $A$ " ${ }^{6}$ В этих терминах систематически интерпретируются логические связки, например, задача $A \rightarrow B$ формулируется следуюшим образом: “по данному решению $A$ найти решение $B$ ". При такой интерпретации выполняются аксиомы исчисления Колмогорова. Однако аксиома $\neg A \rightarrow(A \rightarrow B)$ остается сомнительной. Колмогоров принимает ее в качестве верной на следующих основаниях:

"Что касается задачи 4.1 [т.е. $\neg A \rightarrow(A \rightarrow B)$ ], то это - случай, в котором если $\neg$ р решено, то решение $A$ невозможсно и задача $A \rightarrow B$ не имеет содержания" [26; с. 62]. “Доказательство бессодержательности задачи будет всегда в дальнейшем рассматриваться как ее решение" [26; с. 59].

Таким образом, принцип противоречия принимается в силу соглашения. ${ }^{7}$

В последующие годы Гейтинг постепенно сделал свое определение более точным. В [21] он сказал, что "предложсение выражсат задачу, или, еще лучше, некоторое ожидание". Брауэрово подтверждение предложения $A$ имеет тогда следуюший вид " $A$ можно показать". "Показать" берется здесь в своем неформальном, открытом смысле: построение, устанавливаюшее $A$. Таким образом, $\neg A$, например, есть ожидание того, что $A$ можно привести к противоречию. Что, с современной точки зрения,

\footnotetext{
${ }^{5}[26]$. Статья была представлена 6 февраля 1931 г.

6 Заметим, что под "решением" понимается "положительное решение". В своем знаменитом списке проблем Гильберт (ср. с [9; c. 7]) говорит о "точном решении каждой математической задачи либо в форме действительного ответа на заданньй вопрос, либо путем доказательства невозможности его решения и, таким образом, необходимого провала всех попыток" и, несколькими строчками ниже, утверждает: "Есть задача. Нужно найти ее решение. Вы можете найти его по простой причине, согласно которой в математике нет никакого ignorabimus [не будем знать. Прим. перев.]". Так что здесь слово "решение" используется как "разрешение".

${ }^{7}$ См. также [2; c. 25].
} 
пропушено в формулировках Колмогорова и Гейтинга, так это равномерность, требуемая импликацией. Первые определения были, так сказать, эллиптическими формулировками более точного варианта. Эта равномерность присутствует уже в письме Гейтинга Фрейденталю 1930 года, где он пишет: "Из Ваших замечаний мне стало ясно, что нельзя утверждать, что $A \rightarrow B$ объясняется простьм 'если я полагаю, что $A$, то я должен полагать, что $B$ '; эта идея, и правда, слишком неясна, чтобы вообше служить основой логики. Но и Ваша формулировка: 'если $A$ доказано, то и $B$ доказано' не вполне удовлетворительна; если я спрошу себя, что Вы могли иметь при этом в виду, то, я полагаю, $A \rightarrow B$, как и отрицание, должно учитывать процедуру доказательства: 'у меня есть построение, которое для каждого доказательства $A$ вьводит доказательство $B^{\prime \prime \prime} .8$

В его книге "Mathematische Grundlagenforschung. Intuitionism. Beweistheorie" $[22 ;$ гл. I, § 5.2] современное определение впервые появляется в печати: "Доказательство предложения состоит в реализации требуемого им построения. $A \rightarrow B$ означает концепиию построения, которое каждое доказательство $A$ преобразует в доказательство В".

Колмогоров никогда не возврашался к интуиционистской логике и проблеме правила противоречия. С другой стороны, Гейтинг уделил значительную часть своей карьеры изучению и уточнению интуиционистской логики. Он явным образом вернулся к принципу противоречия в своей книге "Intuitionism. An Introduction", где он прокомметировал роль аксиомы $\neg P \rightarrow(P \rightarrow Q) .{ }^{9}$ Он отмечает, что роль этой аксиомы может казаться не вполне ясной. "Собственно говоря, это добавляет точности определению импликации. Как вы помните, мы можем утверждать, что $P \rightarrow Q$, тогда и только тогда, когда мы располагаем построением, которое, будучи присоединенным к построению $P$, доказьвало бы $Q$. Предположим теперь, что $\vdash \neg P$, т.е. мы вывели противоречие из предположения, что $P$ могло бы быть осуществлено. Тогда, в некотором смысле, это можно рассматривать как построение, которое, будучи присоединенным к доказательству $P$ (которое не может существовать), приводит к доказательству $Q$. Тогда я буду интерпретировать импликации в этом более широком смысле". Здесь видно, что Гейтинг понимал, что в случае ложного антецедента построение интерпретации сомнительно. Это представляет, так сказать, особенность в области импликации. И Гейтинг, более или менее личным вмешательством, восполнил пробел указанным вьше способом. На самом деле он сделал вывод, который неявно содержится в понятии конструктивной интерпретации символа $\rightarrow$. А именно, интерпретация импликации, основанная на обязательстве, неявно заключенном в понятии "построения", преврашается в следуюшее: $p$ является доказательством импликации $A \rightarrow B$ (что кратко выражается символом $p: A \rightarrow B$ ), если для любого $a$ такого, что $a: A$, имеем $p(a): B$. Другими словами, $p$ содержит обешание: как только мне представят доказательство $a$ для $A$, я применю $p$ к $a$ и произведу (за конечное время) доказательство $p(a)$ для $B$. Таким образом, бремя роли импликации в случаe Ex Falso (ложной посылки) приходится на построение. Так как falsum [ложное. Прим. перев.], $\perp$, не имеет доказательства, то любое построение $p$ устанавливает, что $\perp \rightarrow A$ и, конечно, также и $A \rightarrow(\neg A \rightarrow B)$. Таким образом, гейтингово оправда-

\footnotetext{
${ }^{8}$ Письмо Гейтинга Фрейденталю от 25 октября 1930 г. [33].

${ }^{9}[24 ;$ раздел 7.1 .3$]$
} 
ние принципа противоречия является, хотя и с сомнениями, стандартным аргументом на сегодня. Но заслуживает внимания, что это - также и единственньй случай, где явно используется "обещающий” характер построения и где никакого построения не проводится. ${ }^{10}$

Имея в виду этот современный взгляд на импликацию, мы можем оглянуться на замечания Брауэра 1907 года. Как кажется, Брауэра заманила в чрезмерно точное чтение его конструктивной импликации его исходная мотивация скачка от целей к средствам. В это время он считал реальньй переход от $A$ к $B$ сушественным, и на языке интерпретации доказательств он настаивал, что не только дан алгоритм перехода от $A$ к $B$, но также и построение должно быть действительно проведено. В отсутствие последуюших комментариев, мы можем без опаски предполагать, что в дальнейшем он принял более либеральную точку зрения. Однако факт остается фактом - нельзя найти применения принципа противоречия в его сочинениях. Не следует придавать этому слишком большого значения - после этого в литературе о математической истине упорно искали бы пример такого применения.

Возражения против правила противоречия появились снова в 1937 году у Фрейденталя в его “Zur intuitionistischen Deutung logischer Formeln" [16] ${ }^{11}$. Он возражал интерпретации Гейтинга-Колмогорова на основе своего прочтения принщипов Брауэра:

“Так как любое математическое доказательство (интуиционистски) является построением либо ab ovo [с самого начала. - Прим. перев.], либо на основании данного строительного материала (и тогда к конще конщов снова ab ovo, поскольку строительный материал может быть представлен только в виде инструкщий по строительству), то вывод доказательства $B$ из доказательства $A$, или редукиия решения $B$ к решению $A$, должно рассматривать как доказательство $B$, которое снабжается доказательством $A$ по пути".

Это - верное толкование исходного брауэровского наблюдения о гипотетических суждениях, и Фрейденталь обосновывает свои взгляды, принимая очень жесткую конструктивную точку зрения: "Напомним, что правильной формулировкой теоремы является ее доказательство; тогда мы сразу видим, что вывод доказательства $B$ из доказательства $A$, или редукция решения $B$ к решению $A$, возможны, только если показано, что $A$ верно, - и в этом случае импликация теряет всякое значение". Зашишаемый здесь взгляд является максимально жестким, т.е. то, что традиционно назьвалось теоремой, есть не что иное, как последняя строка доказательства (или резюме доказательства). Можно подумать, например, о доказательстве в генщеновском исчислении секвенций. Нижняя строчка является сводкой для упрошенного напоминания, но настоящей теоремой является полное дерево вывода. Если настаивать именно на этом формате теорем и желать закончить вывод на $B$ в случае импликации $A \rightarrow B$, то действительно нужно по пути дать доказательство $A$.

Какие бы достоинства такой подход ни имел, он бы серьезно противоречил математической практике. Цитированный вьше принцип Гейтинга не вполне опровергает Фрейденталя, поскольку нет причин отвергать такие ультражесткие интерпретации;

\footnotetext{
${ }^{10}$ См. также [2; с. 24,25$]$ о гейтинговом выходе из положения.

${ }^{11}$ Представлена в 1934 году.
} 
но интерпретация доказательств по Гейтингу с объектами доказательств совершенно конструктивна. Кроме того, подход Фрейденталя имеет важньй недостаток по сравнению с БГК-интерпретацией; если рассматривать доказательство импликации как содержащее доказательство предположения в дополнение к шагу от $A$ к $B$, то появляется опасность утраты равномерности, придется принять риск предоставления части доказательства, касающейся импликации, снова и снова в каждом новом случае. Это не так для БГК-интерпретации, где импликационная часть сконструирована заранее и может применяться во всех будуших случаях.

Брауэр не принимал участия в дискуссии по крайней мере в печати. Из того факта, что он похвалил работу Гейтинга и представил ее к публикации ${ }^{12}$, можно заключить, что он разделял взгляды Гейтинга на интерпретацию доказательств и, в частности, его следствия для правила противоречия.

Интерпретащиями Колмогорова и Гейтинга занимались многие логики. В частности, так называемые теории типов и интерпретации теорий типов сделаны по образцу интерпретации доказательств, см. [3], [17], [28], [19]. Образное расширение интерпретации доказательств, в сочетании с гёделевской модальной интерпретацией и логикой доказуемости, было введено Артемовьм в [1].

Я признателен Марку ван Аттену и Джону Кюйперу за обсуждения БГК-интерпретации и правила противоречия.

\section{СПИСОК ЛИТЕРАТУРЫ}

[1] S. N. Artemov. Explicit provability and constructive semantics // Bull. Symbolic Logic. 2001. V. 7. №1. P. 1-36.

[2] M. van Atten. On Brouwer. Belmont: Wadsworth, 2004.

[3] H.P. Barendregt. Lambda calculi with types // Handbook of Logic in Computer Science. V. 2 / ed. S. Abramsky et al. Oxford: Oxford Univ. Press, 1992. P. 117-309.

[4] J. M. Borwein. Brouwer-Heyting sequences converge // Math. Intelligencer. 1998. V. 20. № 1. P. $14-15$.

[5] L. E. J. Brouwer. Over de grondslagen der wiskunde // PhD Thesis. Amsterdam, 1907. (Reprinted with Brouwer's corrections and emendations in: D. van Dalen. L. E. J. Brouwer en de grondslagen van de wiskunde. Utrecht: Epsilon, 2001.)

[6] L. E. J. Brouwer. Begründung der Mengenlehre unabhängig vom logischen Satz vom ausgeschlossenen Dritten. Erster Teil, Allgemeine Mengenlehre. Zweiter Teil, Theorie der Punktmengen // Verhandelingen der Koninklijke Akademie van Wetenschappen te Amsterdam 1918. V. 5. P. 1-43; 1919. V. 7. P. 1-33.

[7] L. E. J. Brouwer. Besitzt jede reelle Zahl eine Dezimalbruch-Entwickelung? // Math. Ann. 1921. V. 83. P. 201-210.

[8] L. E. J. Brouwer. Intuitionistishe Zerlegung mathematischer Grundbegriffe // Jahresber. Deutsch. Math.-Verein. 1925 (Dutch version, 1923). V. 33. P. 251-256.

[9] F. Browder (ed.). Mathematical Developments Arising from Hilbert's Problems. Providence, RI: Amer. Math. Soc., 1976. (Proc. Sympos. Pure Math. V. 28.)

[10] D. van Dalen. Logic and Structure. Berlin: Springer-Verlag, 1994.

[11] D. van Dalen. From a Brouwerian point of view. Perspectives on Intuitionism // Philos. Math. (3). 1998. V. 6. № 2. P. 209-226.

[12] D. van Dalen. Intuitionistic logic // Handbook of Philosophical Logic / ed. D. M. Gabbay, F. Guenthner. V. 5. Dordrecht: Kluwer, 2002. P. 1-114.

\footnotetext{
${ }^{12} \mathrm{~B}$ Mathematische Annalen. Но после конфиликта с Annalen Гейтинг забрал обратно свою статью по просьбе Брауэра и опубликовал ее в трудах Прусской Академии.
} 
[13] D. van Dalen, A.S. Troelstra. Constructivism in Mathematics. V. 1, 2. Amsterdam: North-Holland, 1988.

[14] А. Г. Драгалин. Математический интуиционизм. Введение в теорию доказательств. М.: Наука, 1979.

[15] M. Dummett. Elements of Intuitionism. Clarendon: Oxford Univ. Press, 1977; 2-ed. 2000.

[16] H. Freudenthal. Zur intuitionistischen Deutung logischer Formeln // Compositio Math. 1936. V. 4. P. 112-116.

[17] J.- Y. Girard, P. Taylor, Y. Lafont. Proofs and Types. Cambridge: Cambridge Univ. Press, 1989.

[18] V. Glivenko. Sur quelques points de la logique de M. Brouwer // Acad. Roy. Belg. Bull. Cl. Sci. 1929. V. 15. P. 183-188.

[19] Ph. de Groote (ed.). The Curry-Howard Isomorphism. Louvain-la-Neuve: AcademiaErasme, 1995.

[20] A. Heyting. Die formalen Regeln der intuitionistischen Mathematik. I // Sitzungsber. Preuss. Akad. Wiss. Phys.-Math. Kl. 1930. V. 999. P. 52-56.

[21] A. Heyting. Sur la logique intuitionniste // Bull. Acad. Bruxelles (5). 1930. V. 16. P. 957-963.

[22] A. Heyting. Mathematische Grundlagenforschung. Intuitionismus. Beweistheorie. Berlin: Springer-Verlag, 1934. (Рус. пер.: А. Гейтинг. Обзор исследований по основаниям математики. Интуиционизм. Теория доказательства. М.-Л.: ОНТИ, 1936.)

[23] A. Heyting. Bemerkungen zu dem Aufsatz von Herrn Freudenthal "Zur intuitionistischen Deutung logischer Formeln" // Compositio Math. 1936. V. 4. P. 117-118.

[24] A. Heyting. Intuitionism. An Introduction. Amsterdam: North-Holland, 1956. (Pус. пер.: А. Гейтинг. Интуиционизм. Введение. М.: Мир, 1965.)

[25] А. Н. Колмогоров. О принципе tertium non datur // Матем. сб. 1925. Т. 32. № 4. C. $646-667$.

[26] A. N. Kolmogorov. Zur Deutung der intuitionistischen Logik // Math. Z. 1932. V. 35. № 1. Р. 58-65. (Рус. пер.: К толкованию интуиционистской логики // А. Н. Колмогоров. Избранные труды. Математика и механика. М.: Наука, 1985. С. 142-148.)

[27] А. Н. Колмогоров. Письма А. Н. Колмогорова Гейтингу / Пер. и коммент. В. Е. Плиско // УМH. 1988. Т. 43. №6. С. $75-77$.

[28] P. Martin-Löf. Intuitionistic Type Theory. Napoli: Bibliopolis, 1984.

[29] Ю. Т. Медведев. Степени трудности массовых проблем // Докл. АН СССР. 1955. Т. 104. № 4. C. $501-504$.

[30] Ю. Т. Медведев. Финитные задачи // Докл. АН СССР. 1962. Т. 142. № 5. С. 1015-1018.

[31] В.Е. Плиско. Исчисление А.Н. Колмогорова как фрагмент минималшного исчисления // УМH. 1988. Т. 43. №6. С. 79-91.

[32] G. Sundholm. Constructions, proofs and the meaning of the logical constants // J. Philos. Logic. 1983. V. 12. № 2. P. 151-172.

[33] A.S. Troelstra. Logic in the writings of Brouwer and Heyting // Atti del Convegno Internazionale di Storia della Logica (San Gimignano, 1982) / ed. V.M. Abrusci, E. Casari, M. Mugnai. Bologna: Coop. Libraria Universitaria Editrice Bologna, 1983. P. 193-210.

[34] V. A. Uspensky. Kolmogorov and mathematical logic // J. Symbolic Logic. 1992. V. 57. № 2. P. 385-412.

[35] В. А. Успенский, В.Е. Плиско. Интуиционистская логика // А.Н.Колмогоров. Избранные труды. Математика и механика. М.: Наука, 1985. С. 394-404. 